\title{
Functional Brain Imaging and Human Brain Function
}

\author{
Marcus E. Raichle \\ Washington University School of Medicine, St. Louis, Missouri 63110
}

\section{Introduction}

It has been nearly 30 years since the introduction of x-ray computed tomography (CT) set in motion a revolution in medical imaging that changed forever the practice of medicine. The introduction of CT also proved to be an immediate and powerful catalyst for the development of other imaging techniques, particularly positron emission tomography (PET) and magnetic resonance imaging (MRI) [for historical perspectives see Webb (1990) and Kevles (1997)]. With the development of PET and MRI came the opportunity to not only look noninvasively at the anatomy of organs within the living human but also to evaluate their function (Raichle, 2000).

With these new imaging techniques, researchers interested in the function of the human brain were presented with an unprecedented opportunity to examine the neurobiological correlates of human behaviors. This opportunity along with prescient early support from the combined resources of the James S. McDonnell Foundation and the Pew Charitable Trusts contributed significantly to the development of the field of cognitive neuroscience, a field of research that combines the experimental strategies of psychology with various techniques to actually examine how brain function supports mental activities.

The field of cognitive neuroscience, particularly related to studies involving functional imaging techniques, has experienced explosive growth over the past 10 years. This is exemplified not only by a plethora of published papers in established as well as new journals, some devoted exclusively to imaging (e.g., NeuroImage, Human Brain Mapping), and increasing numbers of abstracts at scientific meetings (e.g., well over 300 presentations were made at the past two meetings of the Society For Neuroscience in which "functional MRI" or "fMRI" appeared in the title), but also by the formation of new societies (e.g., the Organization For Human Brain Mapping and the Cognitive Neuroscience Society) and an increasing number of faculty appointments specifically targeting cognitive neuroscience. Equally remarkable has been a worldwide movement to establish research-imaging centers in which expensive imaging equipment (primarily MRI), along with teams of investigators, is devoted exclusively to research. At the present time, with university, governmental, private, and other support (e.g., the Wellcome Trust in the United Kingdom and the Max Planck Society in Germany), there are at least 58 such centers (see Appendix). This development breaks with a longstanding tradition in which research on humans used clinical equipment, part-time, in a hospital setting, usually "after hours."

The subject matter of these developments has been generally well received by the scientific community and the general public. This relates not only to the scientific importance of the work itself

Correspondence should be addressed to Marcus E. Raichle, Washington University School of Medicine, St. Louis, MO 63110.E-mail:marc@npg.wustl.edu.

Copyright $\odot 2003$ Society for Neuroscience $\quad$ 0270-6474/03/233959-04\$15.00/0 but also to the fact that the subject matter of cognitive neuroscience touches on subjects of importance to everyone (e.g., normal as well as disordered memory, attention, language, motivation, emotion, decision making, and even consciousness). In addition, the imaging data produced by cognitive neuroscientists are often quite intriguing; observing the brain of another human at work seems to fascinate scientists and nonscientists alike.

Despite these successes, some researchers have questioned the ability of this approach to provide analyses of brain function that are sufficiently refined to truly enlighten us about the relationship between human behavior and brain function (Nichols and Newsome, 1999). One of the keys to evaluating such concerns is the ability to relate work in cognitive neuroscience and imaging to that which parallels it in other areas of neuroscience.

Among the most important questions are how to relate functional imaging to the cell biology and neurophysiology of brain cells and their microvasculature. Additionally, it seems like a good time to ask whether cognitive neuroscience with its imaging tools has provided us with new insights into brain function and organization or merely confirmed what we have known all along. It was with such issues in mind that we undertook the organization of this miniseries for the Journal, hoping to provide interested readers a perspective on where we are and where we are going in this burgeoning field of research.

This miniseries is composed of six papers. They cannot be expected to provide an exhaustive review of all that is new and important. The field of cognitive neuroscience is already too large and moving to fast to permit that. Rather, these papers were solicited because they provide important evidence, in a number of areas, which is relevant to an understanding of cognitive neuroscience and functional brain imaging in humans. Before turning to some brief introductory comments about the papers that make up this miniseries, some general remarks about functional imaging seem appropriate.

It is useful to consider the intended goal of functional brain imaging. This may seem self-evident to most, yet interpretations frequently stated or implied about functional imaging data $(\mathrm{Ni}-$ chols and Newsome, 1999) suggest that, if we are not careful, functional brain imaging could be viewed as no more than a modern and extraordinarily expensive version of 19th century phrenology. (It is worth noting that functional imaging researchers themselves are occasionally guilty of perpetuating this notion. Data presentations in journal articles, at meetings, and in lay publications often tend to focus on specific areas of the brain, frequently without presenting the entire data set for inspection. These areas are then discussed in terms of complex mental functions_-just what the phrenologists did!).

It is Korbinian Brodmann, one of the pioneers of cytoarchitectonic parcellation of the cerebral cortex, whose perspective I find appealing even though it was written well in advance of the discovery of modern imaging technology (1909 to be exact) (Brodmann, 1909). He said “... Indeed, recently theories have 
abounded which, like phrenology, attempt to localize complex mental activity such as memory, will, fantasy, intelligence or spatial qualities such as appreciation of shape and position to circumscribed cortical zones." He went on to say "... . these mental faculties are notions used to designate extraordinarily involved complexes of mental functions. . . one cannot think of their taking place in any other way than through an infinitely complex and involved interaction and cooperation of numerous elementary activities. . . in each particular case (these) supposed elementary functional loci are active in differing numbers, in differing degrees and in differing combinations. . . Such activities are. .. always the result. . of the function of a large number of suborgans distributed more or less widely over the cortical surface. ..." [for these English translations see Garey (1994), pages 254-255].

With this prescient admonition in mind, the task of functional brain imaging becomes clear: identify multiple regions and their temporal relationships associated with the performance of a well designed task. The brain instantiation of the task will emerge from an understanding of the elementary operations performed within such a network. The great strength of functional brain imaging is that it can contribute uniquely to such a task by providing a broad and detailed view of the processing architecture of cognitively engaged networks. Importantly, this can be accomplished in the brain of most interest to us, the human brain.

In reading the papers in this miniseries it will be important for the reader to note that here as well as elsewhere, imaging data are rich in content as reflected in complex spatial and temporal patterns of activity changes (both increases and decreases) that underlie even the most constrained behaviors of interest to cognitive neuroscientists (hardly the picture portrayed by the early phrenologists). Unraveling the elementary operations instantiated in such networks will be a challenge for all levels of neuroscience. It is fair to say that functional brain imaging, using increasingly sophisticated experimental and analytical strategies and ever more powerful imaging devices, will contribute significantly to this important enterprise in studies of humans as well as experimental animals.

A second general point relates to the nature of the functional imaging signal. It should be recalled that functional brain imaging with PET as well as $\mathrm{PMRI}$ is based on a remarkably consistent relationship between regional changes in the cellular activity of the brain and changes in the circulation and metabolism of that region. Scientists have known that local blood flow in the brain changes in parallel with changes in cellular activity since the late 1800s (Raichle, 1998, 2000). More surprisingly, it was discovered in the 1980s with PET that these changes in blood flow are not accompanied by comparable changes in local oxygen consumption (Fox and Raichle, 1986; Fox et al., 1988). This discrepancy between changes in blood flow and changes in oxygen consumption results in changes in the local concentration of oxygen in the microcirculation of the brain. Because MRI signals are sensitive to the oxygenation of blood (Ogawa et al., 1990a,b), this discovery paved the way for the introduction of fMRI (Bandettini et al., 1992; Kwong et al., 1992; Ogawa et al., 1992).

Much discussion and debate have centered on the cellular events underlying this apparent change in brain metabolism (i.e., a relative increase in glycolysis) [for overview, see Raichle (1998); see also Buxton and Frank (1997) and Buxton et al. (1998)]. Presently, the most parsimonious explanation for this observation is that the increase in glycolysis is related to metabolic changes in astrocytes associated with increased clearance of glutamate from the synapse (Magistretti et al., 1999; Mintun et al., 2001; Shulman et al., 2001). It has been suggested recently that the astrocyte is also a critical link between neurons and blood vessels in orchestrating the changes in blood flow associated with changes in neuronal activity (Zonta et al., 2003). Present information thus leaves little doubt about the central importance of the astrocyte in the cell biology of functional brain imaging signals.

Regardless of how the energy consumption of the brain is altered locally to meet its changing demands, it is critically important to know from a neurophysiological perspective just what cellular events are associated with the local changes in blood flow, metabolism, and tissue oxygenation. To many it may seem obvious that these changes must relate to the spiking activity of neurons because that is what neurophysiologists most commonly measure in relation to behavior. In fact, the spiking activity of neurons has been used as the gold standard in accessing the ability of functional imaging signals to track events of interest within the brain (Hyder et al., 2003; Smith et al., 2003).

The first two papers in this miniseries, one by Logothetis (2003) and the other by Lauritzen and Gold (2003), have much that is new and important to say about how blood flow and other imaging signals relate to the cellular neurophysiology of the brain. Surprisingly for some, it is not the spiking activity of neurons that is important. Rather, synaptic events, as reflected in local field potentials, are most influential in determining the signals obtained with functional imaging, a result anticipated by early tissue autoradiographic studies of metabolism (Sharp, 1976; Sharp et al., 1977; Schwartz et al., 1979).

These new findings obviously pose an interesting challenge when interpreting correspondences, or their absence, in the results of functional imaging and cellular neurophysiology research. The second two papers of this miniseries provide a look at two areas of research in which much evidence does exist for comparison. In the first of these papers, Tootel et al. (2003) provide a detailed review of work in the visual system, probably the best understood of all cortical systems. Fortunately, for the purposes of this comparison, there has been a great deal of imaging work done on the visual system of humans as well. In the second of these papers, Pessoa et al. (2003) review work on attention, a process that has been studied extensively in the visual system by neurophysiologists and, more recently, by imaging researchers.

These two papers make several important points. First, there are many correspondences between neurophysiological results in nonhuman primates and imaging results in humans as reflected in the systems responding to specific stimuli and cognitive manipulations. Second, the neurophysiological and imaging results differ both qualitatively and quantitatively in some instances, inviting interesting hypotheses related not only to possible differences in local brain function being assessed by the two approaches but also to important evolutionary differences between monkeys and humans. Finally, the results from imaging research, particularly with regard to attention, have extended the domain of inquiry well outside of the visual system and to systems in frontal cortex concerned with the control of attention.

The paper by Buckner (2003) on memory addresses one of the most active areas of research in cognitive neuroscience and imaging. The imaging studies of memory (excluding, of course, studies of working memory) emerge from a tradition of memory research that has focused heavily on the hippocampus. Although studies of hippocampal function with imaging have at times been puzzling, studies of the role of the cerebral cortex in memory have been most revealing, as this review suggests. Particularly informative have been results related to the role of the frontal cortex. Although more questions have been raised than answered, it is 
hoped that this work will further stimulate neurophysiologists and others to refocus some of their efforts. Also, as presented in this review, imaging work is providing important clues about the basis for memory deterioration with aging and dementia.

The paper by Burton (2003) on the cortical reorganization associated with blindness provides a dramatic example of the adaptability of the brain to sensory deprivation. Simply put, the visual cortex, despite being deprived of visual input, does not become permanently dormant. Quite to the contrary, it becomes engaged in other tasks. Imaging results of this type should serve as a stimulus to all neuroscientists to probe more deeply into the nature of the underlying processes. Unraveling these processes will not only provide fascinating new insights into brain function and plasticity but may also provide us with a better understanding of how to approach the rehabilitation of the compromised brain.

In closing, it is important to maintain a sense of proportion when it comes to viewing functional imaging signals. In the average adult human, the brain represents $\sim 2 \%$ of the body weight. Remarkably, despite its relatively small size, the brain accounts for $\sim 20 \%$ of the oxygen, and hence calories, consumed by the body (Clark and Sokoloff, 1999), which is 10 times that predicted by its weight alone. In relation to this very high rate of baseline metabolism, functional imaging signals are remarkably small, in metabolic terms usually $<5 \%$ of the ongoing metabolism of the brain, truly modest modulations in ongoing or baseline activity. Evidence now suggests that this baseline activity may instantiate important components of brain function [for an introduction to these issues see Gusnard and Raichle (2001) and Raichle and Gusnard (2002)]. Thus, in our quest to understand brain function we must be careful not focus exclusively on the tip of the iceberg.

Finally, I believe that these papers convey a more general message. As a result of much important new work in neuroscience and the cognitive and social sciences, we can presently say with some confidence that all parties bring important, unique, and complementary perspectives to the task of understanding brain function. No one has a corner on the truth. Working together we have the prospect of progress in one of the last great frontiers of science, understanding the human brain and, in the final analysis, ourselves.

\section{Appendix}

The following 58 institutions possess, or will soon possess, functional brain-imaging equipment (primarily MRI) dedicated exclusively to research. These are found in Belgium (University of Liege), Canada (Clark Institute, Toronto; McGill University/ Montreal Neurological Institute; McMaster University; Robarts Institute, Toronto; Sunnybrooke Hospital, Toronto; University of British Columbia; University of Montreal); Denmark (Aarhus University; University of Copenhagen); France [Service Hospitalier Frederic Joliot (Commissariat à l'Energie Atomique) Orsay; Cyceron (Commissariat à l'Energie Atomique, Institut National de la Santé et de la Recherche Médicale), Caen; Cermep (Institut National de la Santé et de la Recherche Médicale, Centre National de la Recherche Scientifique), Lyons; Neurospin (Commissariat à l'Energie Atomique), Saint-Aubin]; Germany (Juelich Research Institute; Max Planck Institute, Teubingen; Max Planck Institute, Frankfurt; Max Planck Institute, Cologne; Max Planck Institute, Leipzig); Hungary (Debrecen); Italy (Santa Lucia Scientific Institute, Rome; Scientific Institute San Raffaele, Milan); Japan (Akita; Osaka Biosciences Institute; Tohoku University; University of Tokyo); Singapore (University of Singapore); Sweden
(Karolinska Institute; Switzerland: University of Geneva/University of Laussanne); The Netherlands (Donders Center, Nijmegen); UK (Cambridge University; Institute of Neurology, London; Institute of Psychiatry, London; Oxford University); USA (Albany Medical College; Columbia University; Cornell University Medical College; Dartmouth College; Duke University/University of North Carolina; Harvard University; Massachusetts General Hospital/Massachusetts Institute of Technology; National Institutes of Health; New York University; Northwestern University; Princeton University; Rutgers University; Stanford University; University of California, Berkeley; University of California, Los Angeles; University of California, San Diego; University of California, Santa Barbara; University of Michigan; University of Minnesota; University of Oregon; University of Pittsburgh; University of Texas, San Antonia; University of West Virginia; University of Wisconsin; Vanderbilt University; Washington University; Yale University).

\section{References}

Bandettini PA, Wong EC, Hinks RS, Tikofsky RS, Hyde JS (1992) Time course EPI of human brain function during task activation. Magn Reson Med 25:390-397.

Brodmann K (1909) Vergleichende lokalisationlehre der grosshirnrinde. Leipzig: J. A. Barth.

Buckner RL (2003) Functional-anatomic correlates of control processes in memory. J Neurosci 23:000-000.

Burton H (2003) Visual cortex activity in early and late blind people. J Neurosci 23:000-000.

Buxton RB, Frank LR (1997) A model for the coupling between cerebral blood flow and oxygen metabolism during neural stimulation. J Cereb Blood Flow Metab 17:64-72.

Buxton RB, Wong EC, Frank LR (1998) Dynamics of blood flow and oxygenation changes during brain activation: the balloon model. Magn Reson Med 39:855-864.

Clark DD, Sokoloff L (1999) Circulation and energy metabolism of the brain. In: Basic neurochemistry. Molecular, cellular and medical aspects, Ed 6 (Siegel GJ, Agranoff BW, Albers RW, Fisher SK, Uhler MD, eds), pp 637-670. Philadelphia: Lippincott-Raven.

Fox PT, Raichle ME (1986) Focal physiological uncoupling of cerebral blood flow and oxidative metabolism during somatosensory stimulation in human subjects. Proc Natl Acad Sci USA 83:1140-1144.

Fox PT, Raichle ME, Mintun MA, Dence C (1988) Nonoxidative glucose consumption during focal physiologic neural activity. Science 241:462-464.

Garey LJ (1994) Brodmann's localization in the cerebral cortex. London: Smith-Gordon.

Gusnard DA, Raichle ME (2001) Searching for a baseline: functional imaging and the resting human brain. Nat Rev Neurosci 2:685-694.

Hyder F, Rothman DL, Shulman RG (2002) Total neuroenergetics support localized brain activity: implications for the interpretation of fMRI. Proc Natl Acad Sci USA 99:10771-10776.

Kevles BH (1997) Naked to the bone. Medical imaging in the twentieth century. New Brunswick, NJ: Rutgers UP.

Kwong KK, Belliveau JW, Chesler DA, Goldberg IE, Weiskoff RM, Poncelet BP, Kennedy DN, Hoppel BE, Cohen MS, Turner R, Cheng HM, Brady TJ, Rosen BR (1992) Dynamic magnetic resonance imaging of human brain activity during primary sensory stimulation. Proc Natl Acad Sci USA 89:5675-5679.

Lauritzen M, Gold L (2003) Brain function and neurophysiological correlates of signals used in functional imaging. J Neurosci 23:000-000.

Logothetis NK (2003) The underpinnings of the BOLD fMRI signal. J Neurosci 23:000-000.

Magistretti PJ, Pellerin L, Rothman DL, Shulman RG (1999) Energy on demand. Science 283:496-497.

Mintun MA, Lundstrom BN, Snyder AZ, Vlassenko AG, Shulman GL, Raichle ME (2001) Blood flow and oxygen delivery to human brain during functional activity: theoretical modeling and experimental data. Proc Natl Acad Sci USA 98:6859-6864.

Nichols MJ, Newsome WT (1999) The neurobiology of cognition. Nature 402:C35-C38. 
Ogawa S, Lee TM, Kay AR, Tank DW (1990a) Brain magnetic resonance imaging with contrast dependent on blood oxygenation. Proc Natl Acad Sci USA 87:9868-9872.

Ogawa S, Lee TM, Naycik AS, Glynn P (1990b) Oxygenation-sensitive contrast in magnetic resonance imaging of rodent brain at high magnetic fields. Magn Reson Med 16:9-18.

Ogawa S, Tank DW, Menon R, Ellermann JM, Kim S-G, Merkle H, Ugurbil K (1992) Intrinsic signal changes accompanying sensory stimulation: functional brain mapping with magnetic resonance imaging. Proc Natl Acad Sci USA 89:5951-5955.

Pessoa L, Kastner S, Ungerleider LG (2003) Neuroimaging studies of attention: from modulation of sensory processing to top-down control. J Neurosci 23:3987-3995.

Raichle ME (1998) Behind the scenes of functional brain imaging: a historical and physiological perspective. Proc Natl Acad Sci USA 95:765-772.

Raichle ME (2000) A brief history of human functional brain mapping. In: Brain mapping. The systems. (Toga AW, Mazziotta JC, eds), pp 33-75. San Diego: Academic.

Raichle ME, Gusnard DA (2002) Appraising the brain's energy budget. Proc Natl Acad Sci USA 99:10237-10239.

Schwartz WJ, Smith CB, Davidsen L, Savaki H, Sokoloff L, Mata M, Fink DJ,
Gainer H (1979) Metabolic mapping of functional activity in the hypothalamo-neurohypophysial system of the rat. Science 205:723-725.

Sharp FR (1976) Relative cerebral glucose uptake of neuronal perikarya and neuropil determined with 2-deoxyglucose in resting and swimming rat. Brain Res 110:127-139.

Sharp FR, Kauer JS, Shepherd GM (1977) Laminar analysis of 2-deoxyglucose uptake in olfactory bulb and olfactory cortex of rabbit and rat. J Neurophysiol 40:800-813.

Shulman RG, Hyder F, Rothman DL (2001) Cerebral energetics and the glycogen shunt: neurochemical basis of functional imaging. Proc Natl Acad Sci USA 98:6417-6422.

Smith AJ, Blumenfeld H, Behar KL, Rothman DL, Shulman RG, Hyder F (2002) Cerebral energetics and spiking rate: the neurophysiological basis of fMRI. Proc Natl Acad Sci USA 99:10765-10770.

Tootell RBH, Tsao D, Vanduffel W (2003) Neuroimaging weighs in: humans meet macaques in "primate" visual cortex. J Neurosci 23:3978-3986.

Webb S (1990) From the watching Of shadows. New York: Adam Hilger.

Zonta M, Angulo MC, Gobbo S, Rosengarten B, Hossmann K-A, Pozzan T, Carmignoto G (2003) Neuron-to-astrocyte signaling is central to the dynamic control of brain microcirculation. Nat Neurosci 6:43-50. 